\title{
Schwinger-Keldysh formalism of Lattice Gauge Theories
}

Hirotsugu Fujii, Hiroki Hoshina* and Yoshio Kikukawa

Institute of Physics, the University of Tokyo,

Tokyo 153-8902, Japan

E-mail: hfujiiephys.c.u-tokyo.ac.jp, hoshinalhep1.c.u-tokyo.ac.jp,

kikukawadhep1.c.u-tokvo.ac.jp

We formulate the Schwinger-Keldysh formalism of lattice gauge theories by constructing the appropriate unitary transfer matrices in the forward and backward real-time directions for gaugelink and Wilson-Dirac fields. We can then define spectral functions at a finite lattice spacing and also formulate the linear response theory. For a real scalar field in the weak-coupling limit, the two-point Green's function on closed time lattice path was derived analytically and the spectral function was examined numerically. The result is consistent with the continuum theory.

37th International Symposium on Lattice Field Theory - Lattice2019

16-22 June 2019

Wuhan, China

${ }^{*}$ Speaker. 


\section{Introduction}

The Schwinger-Keldysh (SK) formalism[山, [] is a general framework to treat the real-time dynamics of quantum systems. An initial pure or mixed state is described by the density operator and a correlation function of time-dependent observables in Heisenberg picture is given by the trace formula with the initial density operator inserted, which in turn is expressed by path-integration along the closed time-path. The SK formalism, in principle, can be applied in various situations such as out-of-equilibrium and/or background-dependent processes in quantum field theory. Those includes creation and evolution of quark-gluon plasma in ultra-relativistic heavy-ion collisions, cosmological (inflational) evolution of space-time and matter in the early universe, and so on. Some of these processes are strongly coupled and require a certain non-perturbative treatment of the underlying quantum field theory such as QCD and the Standard Model. It is then desirable to formulate the SK formalism within a non-perturbative framework of quantum field theory.

Recently, the SK formalism was introduced in the framework of lattice field theory for the bosonic scalar and gauge-link fields and was studied by Monte Carlo simulations [ [ 3 , 团]. These bosonic lattice models in the SK formalism were obtained from the original models in the Euclidean / imaginary-time formalism by the simple prescription to replace the lattice spacing of time-direction $a_{0}$ to $\pm i a_{0}$ during the forward and backward real-time evolutions in the periodic time-path. In the Monte Carlo simulations, the complex Langevin and Lefschetz thimble methods were employed, trying to evade the sign problem of the lattice models.

In this talk, inspired by these works, we further develop the SK formalism in the framework of lattice gauge theory. We first examine the SK formalism of the scalar boson theory (the $\lambda \phi^{4}$ theory) on the lattice in detail. We observe that the transfer operators in the forward and backward real-time directions are unitary and Hermitian conjugate to each other, but do not commute with the transfer operator in the imaginary-time direction. We also observe that the spectral functions can be defined at a finite lattice spacing. We then formulate the SK formalism of SU(N) lattice gauge theory by constructing the appropriate unitary transfer operators in the real-time directions for gauge-link and Wilson-Dirac fields. We can then formulate the linear response theory and define Kubo's response functions in the framework of lattice gauge theory.

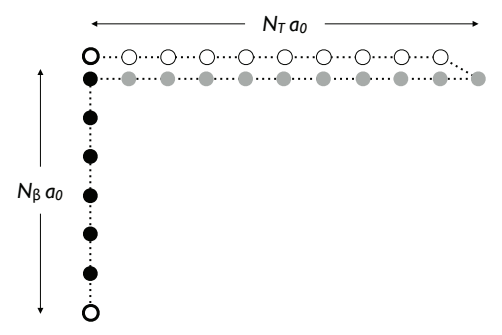

Figure 1: Closed Time Path on a lattice

For simplicity, we consider the case that the initial state is in thermal equilibrium with the inverse temperature $\beta$. Extensions to more general initial states are straightforward. We choose the lattice unit in the three space-like directions and denote the lattice spacing in the time-like direction with $a_{0}$. We assume that the extent of the imaginary-time direction is $N_{\beta} a_{0}(\equiv \beta)$ which defines the inverse temperature, while the extent of the real-time direction is $N_{T} a_{0}(\equiv T)$. The time-like 
lattice coordinate along the closed time path is denoted in general with $s=0, \ldots, 2 N_{T}+N_{\beta}-1$, while the imaginary and real time coordinates are denoted with $\tau=0, \ldots, N_{\beta}-1$, and $t=0, \ldots, N_{T}$, respectively.

\section{Scalar field}

The SK formalism of the lattice scalar model can be defined by the following action[3], 团],

$$
\begin{aligned}
& S_{B / S K}[\phi]=\sum_{s} \sum_{\boldsymbol{x}}\left\{\frac{1}{2 \Delta_{s} a_{0}}(\phi(s+1, \boldsymbol{x})-\boldsymbol{\phi}(s, \boldsymbol{x}))^{2}+a_{0} \frac{\Delta_{s}+\Delta_{s-1}}{2} V[\boldsymbol{\phi}(s, \boldsymbol{x})]\right\}, \\
& V[\boldsymbol{\phi}(s, \boldsymbol{x})]=\phi^{\dagger}(s, \boldsymbol{x})\left[\nabla_{k}^{\dagger} \nabla_{k}+m^{2}\right] \phi(s, \boldsymbol{x})+\frac{\lambda}{2}\left(\phi^{\dagger}(s, \boldsymbol{x}) \phi(s, \boldsymbol{x})\right)^{2},
\end{aligned}
$$

where the time-increment parameter $\Delta_{s}$ is introduced as

$$
\Delta_{s}=\left\{\begin{array}{ll}
+i & \left(s=0, \cdots, N_{T}-1\right) \\
+1 & \left(s=N_{T}, \cdots, N_{T}+N_{\varepsilon}-1\right) \\
-i & \left(s=N_{T}+N_{\varepsilon}, \cdots, 2 N_{T}+N_{\varepsilon}-1\right) \\
+1 & \left(s=2 N_{T}+N_{\varepsilon}, \cdots, 2 N_{T}+N_{\beta}-1\right)
\end{array} .\right.
$$

$N_{\varepsilon}=0$ in []] and $N_{\varepsilon}=N_{\beta} / 2$ in [四]. The partition function of the lattice model is given by

$$
Z_{\mathrm{SK}}[\beta, T] \equiv \int \prod_{s, \boldsymbol{x}} \frac{d \boldsymbol{\phi}(s, \boldsymbol{x})}{\sqrt{2 \pi \Delta_{s} a_{0}}} \mathrm{e}^{-S_{B / S K}[\phi]}=\int \prod_{s=0}^{2 N_{T}+N_{\beta}-1}\left\{\prod_{\boldsymbol{x}} d \boldsymbol{\phi}(s, \boldsymbol{x}) T_{\Delta_{s}}\left[\phi_{s+1}, \phi_{s}\right]\right\}
$$

where the transfer matrices, $T_{\Delta}\left[\phi^{\prime}, \phi\right](\Delta=+i,-i,+1)$, are defined by

$$
T_{\Delta}\left[\boldsymbol{\phi}^{\prime}, \boldsymbol{\phi}\right]=\prod_{\boldsymbol{x}} \frac{1}{\sqrt{2 \pi \Delta a_{0}}} \mathrm{e}^{-\left\{\frac{1}{2 \Delta a_{0}}\left(\boldsymbol{\phi}^{\prime}(\boldsymbol{x})-\phi(\boldsymbol{x})\right)^{2}+\Delta a_{0} \frac{V\left[\phi^{\prime}(\boldsymbol{x})\right]+V[\phi(x)]}{2}\right\}} .
$$

We note that in this formulation the transfer operators, $\hat{T}_{\Delta}(\Delta=+i,-i,+1)$, have the following structure

$$
\hat{T}_{\Delta}=\prod_{x}\left\{\mathrm{e}^{-\Delta a_{0} \frac{V[\hat{\phi}(\boldsymbol{x})]}{2}} \mathrm{e}^{-\Delta a_{0} \frac{\hat{t}(\boldsymbol{x})^{2}}{2}} \mathrm{e}^{-\Delta a_{0} \frac{V[\hat{\phi}(\boldsymbol{x})]}{2}}\right\} \quad(\Delta=+i,-i,+1) .
$$

Then the transfer operators for the real-time evolutions, $\hat{T}_{ \pm i}$, are unitary and Hermite conjugate each other, $\hat{T}_{ \pm i}^{\dagger}=\hat{T}_{ \pm i}{ }^{-1}=\hat{T}_{\mp i}$. These operators $\hat{T}_{ \pm i}$, however, do not commute with the transfer operator for the imaginary-time evolution, $\hat{T}_{+1},\left[\hat{T}_{ \pm i}, \hat{T}_{+1}\right] \neq 0$. It follows that if and only if $N_{\varepsilon}=0$, the partition function $Z[\beta, T]$ is identical to the thermal partition function $Z[\beta]$,

$$
Z[\beta, T]=\operatorname{Tr}\left\{\hat{T}_{+1}^{N_{\beta}} \hat{T}_{-i}^{N_{T}} \hat{T}_{+i}^{N_{T}}\right\}=\operatorname{Tr}\left\{\hat{T}_{+1}^{N_{\beta}}\right\}=Z[\beta] .
$$

It also follows that when $N_{\varepsilon}=0$ the $s$-time-ordered Green's functions have the following properties,

$$
\begin{aligned}
& G^{(n)}\left(\tau_{1}+2 N_{T}, \boldsymbol{x}_{1} ; \cdots ; \tau_{n}+2 N_{T}, \boldsymbol{x}_{n}\right)=G_{\beta}^{(n)}\left(\tau_{1}, \boldsymbol{x}_{1} ; \cdots ; \tau_{n}, \boldsymbol{x}_{n}\right), \\
& G^{(n)}\left(t_{1}, \boldsymbol{x}_{1} ; \cdots ; t_{n}, \boldsymbol{x}_{n}\right)=G^{(n)}\left(2 N_{T}-t_{1}, \boldsymbol{x}_{1} ; \cdots ; t_{n}, \boldsymbol{x}_{n}\right) \quad\left[t_{1}>t_{i}(i \neq 1)\right], \\
& G^{(n)}\left(t_{1}, \boldsymbol{x}_{1} ; \cdots ; t_{n}, \boldsymbol{x}_{n}\right) \neq G^{(n)}\left(t_{1}+t_{0}, \boldsymbol{x}_{1} ; \cdots ; t_{n}+t_{0}, \boldsymbol{x}_{n}\right) .
\end{aligned}
$$


The exact analytic formula of the two-point Green's function in the weak-coupling limit $\lambda=0$, which we denote with $G_{0}\left(s, \boldsymbol{x} ; s^{\prime} \boldsymbol{x}^{\prime}\right)$, can be derived in a block-matrix form as follows:

$$
G_{0}\left(s, s^{\prime} ; \boldsymbol{k}\right)=a_{0}\left(\begin{array}{ll}
\Delta_{F}\left(\tilde{t}, \tilde{t}^{\prime}\right) & \Delta\left(\tilde{t}, \tau^{\prime}\right) \\
\Delta^{T}\left(\tau, \tilde{t}^{\prime}\right) & \Delta_{\beta}\left(\tau, \tau^{\prime}\right)
\end{array}\right)
$$

where $\tilde{t}=0, \cdots, 2 N_{T}-1$ and $\tau=0, \cdots, N_{\beta}-1$, and

$$
\begin{aligned}
& \Delta_{F}\left(\tilde{t}, \tilde{t}^{\prime}\right)=\theta\left(\tilde{t}-\tilde{t}^{\prime}\right) \tilde{\Delta}_{F}\left(\tilde{t}, \tilde{t}^{\prime}\right)+\theta\left(\tilde{t}^{\prime}-\tilde{t}\right) \tilde{\Delta}_{F}\left(\tilde{t}^{\prime}, \tilde{t}\right), \\
& \tilde{\Delta}_{F}\left(\tilde{t}, \tilde{t}^{\prime}\right)=\frac{1}{2 \sinh E\left(1-\mathrm{e}^{-N_{\beta} E}\right)}\left\{\mathrm{e}^{-i E^{\prime} t} \mathrm{e}^{+i E^{\prime} t^{\prime}}\left(\alpha_{+}^{2}+\alpha_{-}^{2} \mathrm{e}^{-N_{\beta} E}\right)\right. \\
& +\mathrm{e}^{-i E^{\prime} t} \mathrm{e}^{-i E^{\prime} t^{\prime}} \alpha_{+} \alpha_{-}\left(1+\mathrm{e}^{-N_{\beta} E}\right) \\
& +\mathrm{e}^{+i E^{\prime} t} \mathrm{e}^{+i E^{\prime} t^{\prime}} \alpha_{+} \alpha_{-}\left(1+\mathrm{e}^{-N_{\beta} E}\right) \\
& \left.+\mathrm{e}^{+i E^{\prime} t} \mathrm{e}^{-i E^{\prime} t^{\prime}}\left(\alpha_{-}^{2}+\alpha_{+}^{2} \mathrm{e}^{-N_{\beta} E}\right)\right\}, \\
& \Delta\left(\tilde{t}, \tau^{\prime}\right)=\frac{1}{2 \sinh E\left(1-\mathrm{e}^{-N_{\beta} E}\right)}\left\{\mathrm{e}^{-i E^{\prime} t} \mathrm{e}^{-E\left(N_{\beta}-\tau^{\prime}\right)} \alpha_{+}+\mathrm{e}^{-i E^{\prime} t} \mathrm{e}^{-E \tau^{\prime}} \alpha_{-}\right. \\
& \left.+\mathrm{e}^{+i E^{\prime} t} \mathrm{e}^{-E\left(N_{\beta}-\tau^{\prime}\right)} \alpha_{-}+\mathrm{e}^{+i E^{\prime} t} \mathrm{e}^{-E \tau^{\prime}} \alpha_{+}\right\}, \\
& \Delta_{\beta}\left(\tau, \tau^{\prime}\right)=\frac{1}{2 \sinh E\left(1-\mathrm{e}^{-N_{\beta} E}\right)}\left\{\mathrm{e}^{-E\left|\tau-\tau^{\prime}\right|}+\mathrm{e}^{-N_{\beta} E} \mathrm{e}^{-E\left|\tau-\tau^{\prime}\right|}\right\}, \\
& \cosh E-1=1-\cos E^{\prime}=\frac{a_{0}^{2}}{2}\left\{\sum_{i} 4 \sin ^{2}\left(k_{i} / 2\right)+m^{2}\right\} \text {, } \\
& \alpha_{ \pm}=\frac{1}{2}\left(1 \pm \frac{\sinh E}{\sin E^{\prime}}\right) \\
& t=\left\{\begin{array}{ll}
\tilde{t} & \left(\tilde{t}=0, \cdots, N_{T}-1\right) \\
2 N_{T}-\tilde{t} & \left(\tilde{t}=N_{T}, \cdots, 2 N_{T}-1\right)
\end{array} .\right.
\end{aligned}
$$

\subsection{Spectral function at a finite lattice spacing}

The spectral function associated with a certain local observable $\hat{O}(t, \boldsymbol{x})$ is defined by the Fourier transformation of the (thermal) expectation value of the four-dimensional commutation relation $\left[\hat{O}(t, \boldsymbol{x}), \hat{O}\left(t^{\prime}, \boldsymbol{x}^{\prime}\right)\right]$. In the SK formalism of the lattice scalar theory, the (thermal) expectation value of the four-dimensional commutation relation can be expressed with the two-point Green's function of $\hat{O}(t, x)$ by the following formula:

$$
\left\langle\left[\hat{O}(t, \boldsymbol{x}), \hat{O}\left(t^{\prime}, \boldsymbol{x}^{\prime}\right)\right]\right\rangle_{\beta}=G_{O}^{(2)}\left(2 N_{T}-t, \boldsymbol{x} ; t^{\prime}, \boldsymbol{x}^{\prime}\right)-G_{O}^{(2)}\left(2 N_{T}-t^{\prime}, \boldsymbol{x}^{\prime} ; t, \boldsymbol{x}\right) .
$$

There is some ambiguity in doing the Fourier transformation with respect to the real-time lattice coordinate $t, t^{\prime}$, because the $t$-time translation invariance does not hold true. We adopt here a symmetric prescription defined as follows:

$$
\rho\left(k_{0}, \boldsymbol{k}\right) \equiv \lim _{N_{T} \rightarrow \infty} a_{0} \sum_{t=0}^{N_{T}} \sum_{\boldsymbol{x}} \mathrm{e}^{i k_{0} a_{0}\left(t-N_{T} / 2\right)} \mathrm{e}^{-i \boldsymbol{k} \cdot \boldsymbol{x}}\left\langle\left[\hat{\boldsymbol{O}}(t, \boldsymbol{x}), \hat{O}\left(N_{T} / 2, \mathbf{0}\right)\right]\right\rangle_{\beta} \quad\left(N_{T} \in 2 \mathbb{Z}\right) .
$$


The spectral function then has the following properties.

$$
\rho\left(k_{0}, \boldsymbol{k}\right)=-\rho\left(-k_{0}, \boldsymbol{k}\right)^{*}, \quad \rho\left(k_{0}, \boldsymbol{k}\right)=\rho\left(k_{0}+2 \pi / a_{0}, \boldsymbol{k}\right) .
$$

Thus the spectral function can be defined with a finite lattice spacing $a_{0}$.

It is instructive to compute the spectral function of $\hat{\phi}(t, x)$ in the weak coupling limit, which we denote with $\rho_{0}\left(k_{0}, \boldsymbol{k}\right)$. Using the result of the two-point Green's function, $G_{0}\left(s, \boldsymbol{x} ; s^{\prime}, \boldsymbol{x}^{\prime}\right)$, obtained in the previous section, the thermal expectation value of $\left[\hat{\boldsymbol{\phi}}(t, \boldsymbol{x}), \hat{\boldsymbol{\phi}}\left(t^{\prime}, \boldsymbol{x}^{\prime}\right)\right]$ is obtained as follows.

$$
\sum_{\boldsymbol{x}} \mathrm{e}^{-i \boldsymbol{k} \cdot \boldsymbol{x}}\left\langle\left[\hat{\boldsymbol{\phi}}(t, \boldsymbol{x}), \hat{\boldsymbol{\phi}}\left(t^{\prime}, \mathbf{0}\right)\right]\right\rangle_{\beta}=a_{0} \frac{\mathrm{e}^{-i E^{\prime}\left(t-t^{\prime}\right)}-\mathrm{e}^{+i E^{\prime}\left(t-t^{\prime}\right)}}{2 \sin E^{\prime}} .
$$

We note that the result is $t$-time translation invariant and independent of the inverse temperature, $\beta=N_{\beta} a_{0}$, as in the continuum theory where the four dimensional commutation $\left[\hat{\boldsymbol{\phi}}(t, \boldsymbol{x}), \hat{\boldsymbol{\phi}}\left(t^{\prime}, \boldsymbol{x}^{\prime}\right)\right]$ is a c-number. The spectral function is then obtained as

$$
\begin{aligned}
\rho_{0}\left(k_{0}, \boldsymbol{k}\right) & =a_{0}^{2} \frac{\pi \delta_{p}\left(k_{0} a_{0}-E^{\prime}\right)}{\sin E^{\prime}}-a_{0}^{2} \frac{\pi \delta_{p}\left(k_{0} a_{0}+E^{\prime}\right)}{\sin E^{\prime}} \\
& =2 \pi \operatorname{sgn}\left(k_{0}\right) \delta\left(\frac{4}{a_{0}^{2}} \sin ^{2}\left(k_{0} a_{0} / 2\right)-\left[\sum_{i} 4 \sin ^{2}\left(k_{i} / 2\right)+m^{2}\right]\right),
\end{aligned}
$$

where $\delta_{p}(\theta)$ is the periodic delta function with the period of $2 \pi$. This is consistent with the result in the continuum theory: $2 \pi \operatorname{sgn}\left(k_{0}\right) \delta\left(k_{0}^{2}-\sum_{i} k_{i}^{2}-m^{2}\right)$.

At a finite $N_{T}$, before taking the limit $N_{T} \rightarrow \infty$, the would-be spectral function is also welldefined as a continuous function of $k_{0}$ and satisfies the properties eqs. (2.201). For the case of $\hat{\phi}(t, \boldsymbol{x})$ in the weak coupling limit, $\rho_{0}\left(k_{0}, \boldsymbol{k} ; N_{T}\right)$ is plotted in figure $\square$.
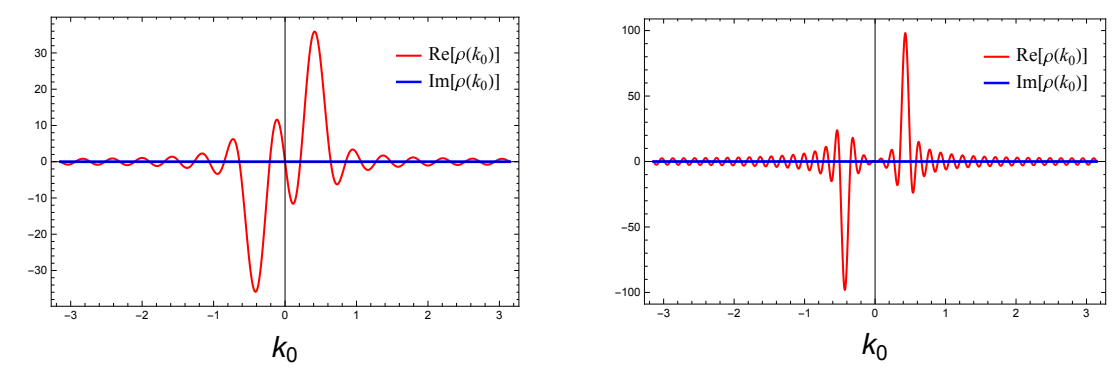

Figure 2: $\rho\left(k_{0}, \boldsymbol{k} ; N_{T}\right)$ for $N_{T}=30$ (left), 80 (right), $N_{\beta}=8, m a_{0}=0, k_{1} a_{0}=0.4267$.

\subsection{A strategy for the SK formalism in the framework of lattice gauge theory}

Based on the above observation about the properties of the SK formalism of lattice scalar theory, let us now discuss our strategy to formulate the SK formalism of QCD-like SU(N) lattice gauge theory with the gauge-link field and the Wilson-Dirac fields. We first require a set of the transfer operators,

$$
\hat{T}_{\Delta} \equiv \hat{T}_{\Delta}^{G} \otimes \hat{T}_{\Delta}^{F} \quad(\Delta=+i,-i,+1),
$$

which satisfy the following three conditions: 
1. First, they are natural extensions of the imaginary-time transfer operator: $\left.\hat{T}_{\Delta}\right|_{\Delta=+1}=\hat{T}_{+1}$.

2. Second, they satisfy the unitarity condition up to certain similarity transformations:

$$
\left.\hat{T}_{\Delta}\right|_{\Delta= \pm i}=\hat{A} \hat{U}_{ \pm i} \hat{A}^{-1}, \quad \hat{U}_{ \pm i}^{\dagger}=\hat{U}_{ \pm i}^{-1}=\hat{U}_{\mp i} .
$$

3. Finally, they commute with the imaginary-time transfer matrices up to higher order corrections in the lattice spacing $a_{0}{ }^{1}:\left[\hat{T}_{+1},\left.\hat{T}_{\Delta}\right|_{\Delta= \pm i}\right]=O\left(a_{0}{ }^{n}\right) \quad\left(n \in \mathbb{Z}_{+}\right)$.

Given such transfer operators, $\hat{T}_{\Delta}(\Delta=+i,-i,+1)$, we next define the partition function by

$$
Z[\beta, T]=\operatorname{Tr}\left\{\hat{T}_{+1}^{\left(N_{\beta}-N_{\varepsilon}\right)} \hat{T}_{-i}^{N_{T}} \hat{T}_{+1}^{N_{\varepsilon}} \hat{T}_{+i}^{N_{T}}\right\}
$$

and then derive the path-integral formula for the partition function (and for the Green's functions),

$$
Z[\beta, T]=\int \prod_{s, \boldsymbol{x}} d U_{\mu}(s, \boldsymbol{x}) \prod_{s, \boldsymbol{x}} d \bar{\psi}(s, \boldsymbol{x}) d \psi(s, \boldsymbol{x}) \mathrm{e}^{-\left\{S_{G / S K}\left[U_{\mu}\right]+S_{F / S K}\left[\psi, \bar{\psi} ; U_{\mu}\right]\right\}} .
$$

We mainly consider the case of $N_{\varepsilon}=0$ for simplicity in the following.

\section{The SK formalism of lattice gauge theory}

The imaginary-time transfer matrix of the $\mathrm{SU}(N)$ link field can be written in the character expansion as follows:

$$
\begin{aligned}
T_{+1}^{G}\left[U^{\prime}, U\right] & =\prod_{\boldsymbol{x}} \mathrm{e}^{-\left\{\sum_{k} \frac{2}{g^{2}} \operatorname{Re} \operatorname{Tr}\left(1-U_{k}^{\prime \dagger}(\boldsymbol{x}) U_{k}(\boldsymbol{x})\right)+\frac{V\left[U^{\prime}(\boldsymbol{x})\right]+V[U(\boldsymbol{x})]}{2}\right\}} \\
& =\prod_{\boldsymbol{x}} \mathrm{e}^{-\frac{1}{2} V\left[U^{\prime}(\boldsymbol{x})\right]} \prod_{k}\left[\sum_{R} d_{R} L_{R}\left(1 / g^{2}\right) \operatorname{Tr}_{R}\left\{U_{k}^{\prime \dagger}(\boldsymbol{x}) U_{k}(\boldsymbol{x})\right\}\right] \mathrm{e}^{-\frac{1}{2} V[U(\boldsymbol{x})]},
\end{aligned}
$$

where $V[U(\boldsymbol{x})]=\sum_{k l} \frac{1}{g^{2}} \operatorname{Re} \operatorname{Tr}\left\{1-U_{k l}(\boldsymbol{x})\right\}$ and $U_{k l}(\boldsymbol{x})$ are the space-like plaquette variables. Then we can introduce the set of the transfer matrices for the gauge-link field $T_{\Delta}^{G}(\Delta=+i,-i,+1)$, which satisfies the three requirements, by

$$
T_{\Delta}^{G}\left[U_{s+1}, U_{s}\right]=\prod_{\boldsymbol{x}} \mathrm{e}^{-\frac{\Delta_{s}}{2} V[U(s+1, \boldsymbol{x})]} \prod_{k}\left[\sum_{R} d_{R} L_{R}\left(1 / g^{2}\right)^{\Delta_{s}} \operatorname{Tr}_{R}\left\{U_{k}^{\dagger}(s+1, \boldsymbol{x}) U_{k}(s, \boldsymbol{x})\right\}\right] \mathrm{e}^{-\frac{\Delta_{s}}{2} V[U(s, \boldsymbol{x})]} .
$$

On the other hand, the imaginary-time transfer matrix of Wilson-Dirac fermion can be written as follows:

$$
\begin{aligned}
T_{+1}^{F}\left[U_{\tau+1}, U_{\tau}\right] & =A_{\tau+1}\left(1-H_{\tau+1} / 2\right) \frac{1}{1+H_{\tau} / 2} A_{\tau}^{-1} \\
A_{\tau} & =B_{\tau}^{-1 / 2}\left[\left(2+a_{0} D_{3 W}\right) \gamma_{0}\right]_{\tau}, \quad B_{\tau}=\delta_{x, x^{\prime}}+a_{0}\left(m_{0} \delta_{x, x^{\prime}}+\sum_{k} \frac{1}{2} \nabla_{k} \nabla_{k}^{\dagger}\right)_{\tau} \\
H_{\tau} & =\gamma_{0} a_{0} D_{3 W \tau} \frac{1}{2+a_{0} D_{3 W \tau}} .
\end{aligned}
$$

\footnotetext{
${ }^{1}$ If the transfer operators for the real-time evolutions were defined using the Hamiltonian of the lattice model, $\hat{H} \equiv-\frac{1}{a_{0}} \ln \hat{T}_{+1}$, the above property would follow immediately: $\hat{T}_{ \pm i}=\mathrm{e}^{\mp i a_{0} \hat{H}} \Rightarrow\left[T_{ \pm i}, T_{+1}\right]=0$. This would be an ideal formulation for the SK formalism in the framework of lattice field theory. However, the explicit or implicit construction of the lattice Hamiltonian $\hat{H}$ is complicated especially for bosonic fields like scalar and gauge link fields.
} 
Then we can introduce the set of the transfer matrices for the Wilson-Dirac field $T_{\Delta}^{F}(\Delta=+i,-i,+1)$, which satisfies the three requirements, by

$$
T_{\Delta}^{F}\left[U_{s+1}, U_{s}\right]=A_{s+1}\left(1-\Delta_{s+1} H_{s+1} / 2\right) \frac{1}{1+\Delta_{s} H_{s} / 2} A_{s}^{-1} .
$$

From these transfer matrices, we can now define the partition function of QCD-like lattice gauge theory in the Schwinger-Keldysh formalism as follows.

$$
\begin{aligned}
Z_{\mathrm{SK}}[\beta, T] & =\operatorname{Tr}\left\{\hat{T}_{+1}{ }^{N_{\beta}} \hat{T}_{-i}{ }^{N_{T}} \hat{T}_{+i}{ }^{N_{T}}\right\} \\
& =\int \mathscr{D} U \mathscr{D} \psi \mathscr{D} \bar{\psi} \mathrm{e}^{-\left\{S_{G / S K}[U]+S_{F / S K}[\psi, \bar{\psi}, U]\right\}} \times \prod_{s=1}^{2 N_{T}}\left\{\operatorname{det} \alpha_{s \Delta_{s}}\right\}^{-1},
\end{aligned}
$$

where $\alpha_{s \Delta_{s}}=\left[\Delta_{s} D_{3 W, s}+B_{s}^{1 / 2} A_{s}\right] / 2$. The actions of the gauge-link field and the Wilson-Dirac fields are given as

$$
\begin{aligned}
& S_{G / S K}=\sum_{s, \boldsymbol{x}}\left\{K_{\Delta_{s}}[U(s, \boldsymbol{x}), U(s+1, \boldsymbol{x})]+\frac{\Delta_{s}+\Delta_{s-1}}{2} V[U(s, \boldsymbol{x})]\right\} \\
& S_{F / S K}=\sum_{n, s, s^{\prime}} \bar{\psi}(s, \boldsymbol{n})\left[-\left(\frac{1-\gamma_{0}}{2}\right) \nabla_{0}-\left(\frac{1+\gamma_{0}}{2}\right) \nabla_{0}^{\dagger}+a_{0} D_{3 W} V_{s, s^{\prime}}\right] \psi\left(s^{\prime}, \boldsymbol{n}\right),
\end{aligned}
$$

where $K_{\Delta_{s}}=-\sum_{k} \ln \left[\sum_{R} d_{R}\left(L_{R}\left(1 / g^{2}\right)\right)^{\Delta_{s}} \operatorname{Tr}_{R}\left\{U_{k}(s) U_{k}^{\dagger}(s+1)\right\}\right]$ and $V_{s, s^{\prime}}$ is defined by

$$
V_{s, s^{\prime}}=\left(\begin{array}{cc}
\frac{1+\Delta_{s}}{2} \delta_{s, s^{\prime}}-\frac{1-\Delta_{s}}{2} \delta_{s, s^{\prime}+1} & 0 \\
0 & \frac{1+\Delta_{s-1}}{2} \delta_{s, s^{\prime}}-\frac{1-\Delta_{s}}{2} \delta_{s+1, s^{\prime}}
\end{array}\right) .
$$

\section{Summary}

We have constructed the real-time transfer matrices for the gauge-link field and the WilsonDirac fields, and formulated the Schwinger-Keldysh formalism of QCD-like lattice gauge theory. It is then possible to define spectral functions non-perturbatively at a finite lattice spacing and to formulate the linear response theory. The authors refer the reader to [6] for detail. For a real scalar field in the weak-coupling limit, the two-point Green's function on closed time lattice path was derived analytically and the spectral function was examined numerically. The result is consistent with the continuum theory.

\section{References}

[1] J. Schwinger, Journal of Mathematical Physics 2, 407 (1961).

[2] L. V. Keldysh, Zh. Eksp. Teor. Fiz. 47, 1515 (1964), [Sov. Phys. JETP20, 1018 (1965)].

[3] J. Berges, S. Borsanyi, D. Sexty and I.-O. Stamatescu, Phys. Rev. D 75, 045007 (2007), [hep-lat/0609058].

[4] A. Alexandru, G. Basar, P. F. Bedaque, S. Vartak and N. C. Warrington, Phys. Rev. Lett. 117, no. 8, 081602 (2016), [arXiv:1605.08040 [hep-lat]].

[5] A. Alexandru, G. Basar, P. F. Bedaque and G. W. Ridgway, Phys. Rev. D 95, no. 11, 114501 (2017), [arXiv:1704.06404 [hep-lat]].

[6] H. Fujii, H. Hoshina, Y. Kikukawa, in preparation. 